\title{
ENTEROPARASITOSES NA POPULAÇÃO INFANTIL, SUA PREVALÊNCIA E OS MODELOS DE DECISÃO UTILIZADOS: REVISÃO SISTEMÁTICA
}

\author{
Ulanna Maria Bastos Cavalcante
}

Mestranda no Programa de Pós-Graduação em Modelos de Decisão e Saúde Universidade Federal da Paraíba (UFPB), Brasil.

E-mail: ulannacavalcante@hotmail.com

\section{Silvia Adelaide Linhares de Melo}

Mestranda no Programa de Pós Graduação em Modelos de Decisão e Saúde Universidade Federal da Paraíba (UFPB), Brasil.

\section{Caliandra Maria Bezerra Luna Lima}

Doutora em Produtos Naturais e Sintéticos Bioativos pela Universidade Federal da Paraíba (UFPB); Docente Adjunta III do Departamento de Fisiologia e Patologia e Docente colaboradora do Programa de Pós-Graduação Interdisciplinar em Modelos de Decisão e Saúde da Universidade Federal da Paraíba (UFPB), Brasil.
RESUMO: Este estudo tem como objetivo realizar uma revisão de literatura sobre a prevalência de enteroparasitoses na população infantil e principais modelos de decisão utilizados na tomada de decisão. Procedeu-se uma revisão sistemática, pela combinação dos descritores "doenças parasitárias", "crianças", "prevalência", "parasitic diseases", "children" e "prevalence", na Biblioteca Virtual de Saúde, nas bases de dados LILACS (Literatura em Ciências da Saúde) e SciELO (Scientific Eletronic Library Online), entre 2010 e 2014. Observouse que os parasitos mais frequentes foram: Ascaris lumbricoides; Ancylostomidae; Trichuris trichiura; Giardia lamblia; Entamoeba coli; Endolimax nana. Em relação ao tipo de análise estatística utilizada, a regressão é a única considerada um modelo de tomada de decisão; as demais são técnicas capazes de gerar resultados. Concluise que Ascaris lumbricóides, Ancylostomidae, Trichuris trichiura, Giardia lamblia, Entamoeba coli e Endolimax nana são os tipos que mais afetam a população. As análises estatísticas estão sendo feitas de forma pouco detalhada, quanto ao seu delineamento.

PALAVRAS-CHAVE: Crianças; Doenças Parasitárias; Prevalência.

\section{ENTEROPARASITOSIS IN CHILDREN, PREVALENCE AND DECISION MODELS EMPLOYED: A SYSTEMATIC REVIEW}

\begin{abstract}
A review of the literature on the prevalence of enteroparasitosis in children and main decision models. A systematic review was undertaken by a set of descriptors: "parasite diseases", "children", "prevalence", in the Health Virtual Library at data bases LILACS (Literature in Health Science) and SciELO (Scientific Eletronic Library Online), between 2010 and 2014. The most frequent parasites were: Ascaris lumbricoides; Ancylostomidae; Trichuris trichiura; Giardia lamblia; Entamoeba coli; Endolimax nana. Regression statistical analysis was the single decision-taking model; all the others were capable of producing results. Results showed that Ascaris lumbricoides; Ancylostomidae; Trichuris trichiura; Giardia lamblia; Entamoeba coli; Endolimax nana are parasites that affect the children population. Statistical analysis is undertaken with few details with regard to design.
\end{abstract}

KEY WORDS: Diseases by Parasites; Children; Prevalence. 


\section{INTRODUÇÃO}

A saúde pública no Brasil, e nos demais países em desenvolvimento, tem como um dos seus graves problemas as enteroparasitoses, doenças cujos agentes etiológicos são helmintos ou protozoários. Estas doenças tendem a ocorrer de acordo com as condições de saneamento básico, nível socioeconômico, grau de escolaridade, idade e hábitos de higiene, entre outras variáveis (BASSO et al., 2008). Elas estão entre as Doenças Negligenciadas (DN) e são tidas como um conjunto de doenças causadas por agentes infecto-parasitários, que são responsáveis por produzirem relevantes danos à saúde, sendo eles: físico, cognitivo e socioeconômico em crianças e adolescentes, principalmente na população mais desfavorecida (MATHERS et al., 2012).

As parasitoses intestinais apresentam uma elevada magnitude e ampla distribuição geográfica; estas características aliadas às repercussões negativas que podem causar no organismo humano têm conferido a essas infecções uma posição relevante entre os principais problemas de saúde da população (FONSECA et al., 2010). Dentre os grupos etários que têm maior vulnerabilidade à contaminação por parasitos intestinais, encontra-se o das crianças, uma vez que, geralmente, não realizam medidas de higiene pessoal de forma adequada e, frequentemente, se expõem ao solo e à água, que são importantes focos de contaminação (MONTRESOR et al., 2002).

Apesar de apresentarem baixa taxa de mortalidade, as parasitoses são responsáveis por altos índices de morbidade, principalmente nos países em desenvolvimento, onde são utilizadas como indicadores do desenvolvimento socioeconômico (WORLD HEALTH ORGANIZATION, 2014). As infecções por helmintos estão entre as mais comuns em países em desenvolvimento e podem comprometer o estado nutricional, causando hemorragia interna, que pode levar à perda de ferro e anemia; má absorção de nutrientes; diarreia e perda de apetite, que pode conduzir a uma redução no consumo de energia. Infecções por hemiltos também podem causar déficit cognitivo, bem como danos nos tecidos que pode exigir cirurgia corretiva (FREI; JUNCANSEN; RIBEIRO-PAES, 2008).
A análise de regressão é considerada como um dos métodos de análise de dados da teoria estatística mais utilizados na pesquisa científica. A origem do modelo clássico de regressão ocorreu nos trabalhos de astronomia elaborados no período de 1809 a 1821 por Gauss, trata-se de uma técnica adequada para estudar o efeito que variáveis explicativas exercem sobre uma variável resposta (SOUZA, 2011).

A regressão logística tem se constituído em um dos principais métodos de modelagem estatística de dados. Mesmo quando a resposta de interesse não é originalmente do tipo binário, alguns pesquisadores têm dicotomizado a resposta de modo que a probabilidade de sucesso possa ser ajustada através desse modelo. Embora a regressão logística seja conhecida desde os anos 50, foi através de Cox (1970) e Cox e Snell (1989) que ela ficou popular entre os usuários de Estatística.

Os estudos que abordam revisão sistemática são muito importantes para analisar pesquisas desenvolvidas em determinada área do conhecimento, facilitam o acesso para os pesquisadores que precisam de uma rápida revisão, além disso, são capazes de direcionar estudos futuros. Haja vista o cenário apresentado, esta pesquisa traz informação à saúde da população, uma vez que é possível mostrar quais métodos são mais adequados para auxiliarem os órgãos de saúde responsáveis na sua tomada de decisão. O objetivo deste estudo foi realizar uma revisão de literatura sobre a prevalência de enteroparasitoses na população infantil e principais modelos de decisão utilizados na tomada de decisão.

\section{METODOLOGIA}

Para a obtenção dos estudos a serem analisados procedeu-se uma revisão sistemática, a busca foi realizada pela combinação dos descritores "doenças parasitárias", "crianças", "prevalência", "parasitic diseases", "children" e "prevalence", na Biblioteca Virtual de Saúde, nas bases de dados LILACS (Literatura em Ciências da Saúde) e SciELO (Scientific Eletronic Library Online). Os critérios de inclusão dos artigos no presente estudo foram: artigos publicados em português e inglês; entre os anos de $2010 \mathrm{e}$ 2014; com amostras de pacientes infantis e que avaliavam 
a prevalência de enteroparasitoses na população infantil. Foram utilizados os seguintes critérios de exclusão: artigos com amostras de adolescentes, adultos ou idosos com enteroparasitoses e pesquisas não disponibilizadas na íntegra.

Diante dos resultados de cada busca, a seleção inicial ocorreu pela simples leitura dos títulos encontrados, em seguida foram descartados aqueles que não se enquadravam ao tema, local ou período selecionados, bem como os que não continham dados originais. Para os considerados potencialmente elegíveis, avaliou-se os resumos para uma segunda etapa de seleção quanto à elegibilidade. Os artigos que à primeira vista cumpriam com os critérios de inclusão foram obtidos e analisados na íntegra, sendo finalmente incluídos aqueles que contemplavam a proposta da presente revisão sistemática. Adicionalmente, foram examinadas as listas de suas referências bibliográficas para busca de outros artigos, que até então não tinham sidos rastreados pela busca eletrônica. Por fim, com os estudos identificados que cumpriram os critérios de inclusão, foram construídas tabelas com suas características.

\section{RESULTADOS}

Sem considerar os critérios de inclusão e exclusão, encontrou-se inicialmente 239 publicações no idioma português e inglês, na base de dados LILACS. Destas publicações, excluindo-se aquelas que não se encaixavam nos critérios supracitados, restaram 32 artigos. Após leitura dos títulos foram excluídos 07 artigos, restando 25 artigos para leitura. $\mathrm{Na}$ base de dados SciELO, foram encontrados 07 artigos no idioma português e inglês, sendo excluídos 03 por não se encaixarem nos critérios escolhidos.

Finalmente, os artigos selecionados foram comparados nas duas bases de dados, LILACS e SciELO, para verificar quais publicações estavam repetidas. Constatou-se a repetição de 05 artigos. Assim, ao final, 24 artigos foram analisados acerca da prevalência e doenças parasitárias em crianças, bem como os métodos estatísticos usados. O fluxograma de estudos utilizados encontra-se na Tabela 1.

Tabela 1. Estudos Excluídos e Incluídos na Revisão Sistemática, Segundo Critérios Adotados

\begin{tabular}{cccccc}
\hline Base de dados & Artigos Encontrados & Artigos excluídos & Total & Artigos repetidos & Total utilizado \\
\hline LILACS & 32 & 07 & 25 & & \\
SciELO & 07 & 03 & 04 & 05 & 24 \\
\hline
\end{tabular}

Observou-se que a produção científica sobre enteroparasitoses em escolares é vasta e que sua distribuição geográfica e instalação se dão principalmente na população de baixa renda, classe em que há precariedade de saneamento básico e falta de higiene, por exemplo. Dentre os 24 artigos selecionados, 08 não apresentaram descritos o quantitativo de indivíduos por sexo, o que representam $33,33 \%$ do total de artigos. Ainda com referência aos participantes do estudo, as amostras com pacientes parasitados e não parasitados estão dispostas na Tabela 2. Nesses 24 estudos observouse que $7,69 \%$ são internacionais e $92,31 \%$ nacionais. Quanto à região, 34,61\% foram feitos no Nordeste. Os tipos de parasitos mais frequentes em ordem decrescente foram: Ascaris lumbricoides; Ancylostomidae; Trichuris trichiura; Giardia lamblia; Entamoeba coli; Endolimax nana.

Tabela 2. Percentual de Parasitados Entre o Total de Artigos Analisados

\begin{tabular}{lcc}
\hline & N & $\%$ \\
\hline Parasitados & 9 & $36 \%$ \\
Não - parasitados & 15 & $64 \%$ \\
Total & 24 & $100 \%$ \\
\hline
\end{tabular}

No que se refere às revistas que publicam sobre parasitoses intestinais, houve uma diversidade bastante considerável. A Revista de Patologia Tropical foi a que mais apresentou publicações no período considerado, 
com nove artigos publicados, seguida da Revista da Sociedade Brasileira de Medicina Tropical e do Caderno de Saúde Pública, ambas com quatro publicações. Já em relação às demais revistas, foi verificado que resultaram uma publicação cada. No que diz respeito ao ano das publicações analisadas, percebe-se aumento de publicações sobre o tema nos últimos anos, especialmente os anos 2010 e 2012 (Tabela 3).

Tabela 3. Distribuição dos Artigos de Acordo com o Ano de Publicação

\begin{tabular}{lcc}
\hline Ano de publicação & N & $\%$ \\
\hline 2010 & 9 & $37 \%$ \\
2011 & 4 & $17 \%$ \\
2012 & 7 & $29 \%$ \\
2013 & 4 & $17 \%$ \\
2014 & 0 & 0 \\
\hline Total & 24 & $100 \%$ \\
\hline
\end{tabular}

Em relação ao tipo de análise estatística utilizada, pode-se observar na Tabela 4 a sua distribuição. Vale ressaltar que, em alguns estudos, não havia clareza no que se refere ao delineamento do estudo e suas análises estatísticas, porém, a partir das características observadas em seus participantes, dos instrumentos e da análise de dados realizadas, foi possível essa classificação.

Tabela 4. Método de Análise Estatística Usado nos Artigos Analisados

\begin{tabular}{lcc}
\hline Método de análise estatística & N & \% \\
\hline Descritiva & 11 & $46 \%$ \\
Intervalo de Confiança & 6 & $25 \%$ \\
Qui-quadrado & 3 & $13 \%$ \\
Regressão logística & 2 & $8 \%$ \\
Análise espacial & 1 & $4 \%$ \\
Estudo temporal & 1 & $4 \%$ \\
\hline Total & 24 & $100 \%$ \\
\hline
\end{tabular}

\section{DISCUSSÃO}

Este estudo traz que em sua maioria as pessoas são acometidas por parasitos do tipo: Ascaris lumbricoides; Ancylostomidae; Trichuris trichiura; Giardia lamblia; Entamoeba coli; Endolimax nana; estas duas últimas são consideradas enterocomensais, ou seja, não são patogênicos. Contudo, são indicadores da ausência de saneamento básico tornando-o um dado importante para o aprimoramento das políticas públicas de saúde. Corroborando estes dados, Pullan e Brooker (2010) mostram que os parasitos intestinais, do tipo helmintos, encontrados com mais frequência em seres humanos são: Ascaris lumbricoides, Trichuris trichiura e os Ancylostomidae, como Necator americanus e Ancylostoma duodenale, sendo que mais de 05 bilhões de pessoas já estão em risco de transmissão por helmintos através do solo. No que concerne aos protozoários, destacam-se Entamoeba bistolytica e Giardia intestinalis.

A prevalência de parasitados (36\%) mostrou-se menor do que os não-parasitados (64\%). A ocorrência de parasitoses é bastante variável nas diferentes regiões do Brasil, estando relacionada ao desenvolvimento socioeconômico das populações e à metodologia dos estudos realizados. Santos e Merlini (2010), em estudo realizado com a população do município de Santa Helena, Paraná, detectaram uma prevalência com 16\%. Fontes et al. (2003), por sua vez, relataram que $92 \%$ dos escolares de Barra do Santo Antônio, Alagoas, apresentavam diagnósticos positivos.

Com base na análise dos artigos publicados sobre parasitoses intestinais, podemos verificar, primeiramente, que o volume de publicações sobre o assunto é muito vasto, e que ela ocorreu principalmente nos anos de 2010 e 2012. Quando observa-se a parasitose intestinal no seu contexto mais amplo, proposto e sistematizado pela Organização Mundial de Saúde (OMS), tais pesquisas relacionam a parasitose intestinal e o ambiente de vida das pessoas e investigaram diferentes aspectos sociais associados ao problema trazido pela doença ao paciente (FONSECA et al., 2010), como por exemplo, o seu desenvolvimento físico (FILHO et al., 2011; SILVA et al., 2010) e em alguns casos abordam seu tratamento.

Quanto aos métodos de análise estatística utilizados nos artigos estudados, a regressão é a única considerada um modelo de tomada de decisão. Os demais são técnicas capazes de gerar resultados, a exemplo da análise descritiva, intervalo de confiança, análise espacial, mas que não são consideradas modelos 
de decisão, porque não auxiliam na tomada de decisão. Em grande parte dos artigos, os autores referem-se ao valor de $\mathrm{p}$, porém não explicam o significado desse termo, o que não torna claro ao leitor do que se trata. Em resultados observados em 552 artigos, mostraram que entre as metodologias aplicadas, o desvio padrão, a média, a análise de correlação e a análise de regressão foram as mais utilizadas (SOTTO, 2011). De acordo com Grácio e Garrutti (2005), tanto nos artigos como nas pesquisas de pós-graduação, na área de Educação, que têm por finalidade verificar os procedimentos utilizados, constataram que quando os pesquisadores utilizam a metodologia estatística, a maioria é de natureza descritiva.

A presente revisão sistemática apresenta como aspectos positivos a discussão sobre a importância dos métodos de tomada de decisão, para auxiliarem os órgãos de saúde responsáveis, bem como o incentivo de mais publicações utilizando-os. Para isso a educação em saúde entra como um ferramenta que deve ser pensada como um exercício coletivo, buscando novos instrumentos para o trabalho (SILVA et al., 2015). Tem como limitação o fato de haver poucos estudos que usem tais métodos de tomada de decisão para poder avançar tal discussão.

\section{CONCLUSÃO}

A partir do que foi exposto, pode-se concluir que Ascaris lumbricóides, Ancylostomidae, Trichuris trichiura, Giardia lamblia, Entamoeba coli endolimax nana são os tipos de parasitos que mais afetam a população, especialmente as de baixa renda. As análises estatísticas estão sendo feitas de forma pouco detalhada, usando apenas análises descritivas, visto que o seu aprofundamento é relevante aos leitores, em especial, aos tomadores de decisão que em posse dessas informações serão capazes de fundamentar cientificamente suas escolhas. Sendo relevante também definir como tais métodos são usados, quais as suas finalidades e qual o benefício/implicação da sua utilização em cada estudo.

\section{REFERÊNCIAS}

ARAUJO FILHO, H. B. et al. Parasitoses intestinais se associam a menores índices de peso e estatura em escolares de baixo estrato socioeconômico. Rev Paul Pediatr., v. 29, n. 4, p. 521-528, 2011.

BASSO, R. M. C. et al. Evolução da prevalência de parasitoses intestinais em escolares em Caxias do Sul, RS. Rev Soc Bras Med Trop., v. 41, n. 3, p. 263-268, 2008.

COX, D. R. The analysis of binary data. Methuen, London, 1970.

COX, D. R.; SNELL, E. J. The analysis of binary data. 2nd Edition. Chapman and Hall, London, 1989.

FONSECA, E. O. L. et al. Prevalência e fatores associados às geo-helmintíases em crianças residentes em municípios com baixo IDH no Norte e Nordeste brasileiros. Cad Saúde Pública, v. 26, n. 1, p. 143-152, 2010.

FONTES, G. et al. Influência do tratamento específico na prevalência de enteroparasitoses esquistossomose mansônica em escolares do município de Barra de Santo Antônio, AL. Rev Soc Bras Med Trop., v. 36, n. 5, p. 625628, 2003.

FREI, F.; JUNCANSEN, C.; RIBEIRO-PAES, J. T. Levantamento epidemiológico das parasitoses intestinais: viés analítico decorrente do tratamento profilático. Cad Saúde Pública, v. 24, n. 12, p. 2919-2925, 2008.

GRÁCIO, M. C. C.; GARRUTTI, E. A. A. Seleção de conteúdos estatísticos para a educação. Rev Educ Matemática, 2005.

MATHERS, C. D. et al. Global burden of disease in young people aged 10-24 years: authors' reply. Lancet, v. 379, n. 9810 , p. 28, 2012.

MONTRESOR, A. et al. Helminth control in school- age children: a guide for managers of control programmes. Geneva: WHO; 2002.

PULLAN, R. L.; BROOKER, S. J. The global limits and population at risk of soil transmitted helminth infections 2010. Parasit vectors., v. 5, p. 81, 2012.

SANTOS, S. A.; MERLINI, L. S. Prevalence of enteroparasitosis in the population of Maria Helena, Paraná State. Cienc Saude Colet, v. 15, p. 899-905, 2010.

SILVA, B. T. et al. Educação continuada em enfermagem 
no âmbito da educação permanente em saúde: revisão integrativa. Saúde e Pesquisa, v. 8, n. 1, p. 131-140, jan./ abr. 2015.

SILVA, R. R. et al. Prevalence of parasitic diseases and nutritional status of preschool children in municipal educational centers in the south of Minas Gerais state. Nutrire: Rev Soc Bras Alim Nutr J Brazilian Soc Food Nutr., São Paulo, SP, v. 35, n. 1, p. 59-72, abr. 2010.

SOTTO, L. U. A Aplicabilidade dos métodos estatísticos em estudos da área de biologia: análise em periódicos 2010 a 2011. Relatório (Programa de Iniciação Científica) Faculdade de Ciências Exatas e da Natureza, Universidade Metodista de Piracicaba-Piracicaba, 2011.

SOUZA, T. C. Ensaios sobre modelos de regressão com dispersão variável. 2011. Tese. Universidade Federal de Pernambuco, Recife, 2011.

WORLD HEALTH ORGANIZATION. Deworming for health and development. Report of the Third Global Meeting of the Partners for Parasite Control. Geneva: World Health Organization, 2014.

Recebido em: 28 de setembro de 2015 Aceito em: 06 de novembro de 2015 